\title{
EFEITO DA VAZÃO DE GÁS NA QUALIDADE DA MEDIDA DA VELOCIDADE DO LÍQUIDO EM UMA SEÇÃO DE UMA COLUNA DE BOLHAS COM EXPANSÃO DE DIÂMETRO POR PIV.
}

\author{
D. I. SANCHEZ-FORERO ${ }^{1}$, K. K. da COSTA ${ }^{1}$, R. L. AMARAL ${ }^{1}$, O. P. TARANTO ${ }^{1}$, S.S.V. \\ VIANNA $^{1}$, M. MORI ${ }^{1}$ \\ ${ }^{1}$ Universidade Estadual de Campinas, Faculdade de Engenharia Química. \\ E-mails para contato: sanchezd@feq.unicamp.br, val@ feq.unicamp.br, \\ savio@feq.unicamp.br, mori@feq.unicamp.br.
}

\begin{abstract}
RESUMO - A velocimetria por imagem de partícula (PIV) tem demonstrado um bom desempenho na análise de escoamentos de uma única fase. Entretanto, em escoamentos multifásicos encontrados em coluna de bolhas, por exemplo, o acesso óptico da câmera e a iluminação homogênea da luz laser são afetados pela presença das bolhas. Este trabalho tem como objetivo avaliar a qualidade das medidas PIV da fase líquida com o aumento da vazão de gás em uma coluna de bolhas com expansão de diâmetro. Além do melhor ângulo da câmera em relação ao plano de luz laser, foi avaliado o processamento da imagem PIV com o aumento da quantidade de imagem de bolhas na área investigada. Os ensaios experimentais foram realizados no sistema água-ar, em uma coluna cilíndrica de acrílico de $1 \mathrm{~m}$ de altura e diâmetro inferior e superior igual a $145 \mathrm{~mm}$ e $246 \mathrm{~mm}$, respectivamente. A faixa de vazão de gás investigada foi de $2-20 \mathrm{~L} / \mathrm{min}$. A área investigada tem um tamanho de $14,5 \times 10 \mathrm{~cm}$ e se localiza a $15,4 \mathrm{~cm}$ do distribuidor de gás.
\end{abstract}

\section{INTRODUÇÃO}

No estudo de coluna de bolhas é importante analisar os efeitos das condições de operação, propriedades do sistema, e dimensionamento da coluna em seu desempenho. Com o intuito de obter os parâmetros necessários para avaliar estes efeitos, se faz necessário o uso de técnicas de análise visual ou de correlação de imagem (Bröder e Sommerfeld, 2007). A Velocimetria por Imagem de Partícula (PIV) é uma técnica não intrusiva, que utiliza um traçador para tornar o padrão de fluxo visível através de um equipamento de detecção. Esta técnica permite determinar os campos de velocidade instantânea do fluido, através do deslocamento de pequenos grupos de partículas em determinadas áreas de estudo (janelas de interrogação) utilizando funções de correlação (Prasad, 2000). O principal problema neste tipo de medições refere-se à qualidade da imagem gravada e o processamento dessa imagem. Em sistemas multifásicos, especialmente em colunas de bolhas, é comum que existam áreas de maior intensidade de luz, produto de múltiplos fatores, um destes relacionado à presença de bolhas dentro do campo de medição e seu grande espalhamento de luz laser em relação à partícula traçadora. Assim, este trabalho tem por objetivo avaliar a qualidade das medições PIV da fase líquida, variando a vazão volumétrica de gás em uma faixa de 2 - $20 \mathrm{~L} / \mathrm{min}$. 


\section{PROCEDIMENTO EXPERIMENTAL}

Para estudar o efeito da vazão da fase gasosa na qualidade da medida PIV, foram realizados testes em uma coluna feita de acrílico com dois cilindros de diâmetros diferentes, 14,5 e $24,5 \mathrm{~cm}$ (Figura 1a). A coluna possui 1 metro de altura e foi preenchida com água a $25^{\circ} \mathrm{C}$ até uma altura de $80 \mathrm{~cm}$. A fase gasosa utilizada foi ar a $25^{\circ} \mathrm{C}$. A área investigada tem um tamanho de $14,5 \times 10 \mathrm{~cm}$ e se localiza a $15,4 \mathrm{~cm}$ do distribuidor (Figura 1b). O distribuidor é uma placa plana com 37 furos igualmente distribuídos em um arranjo quadrado (Figura 1c). Foram utilizadas esferas de vidro com um diâmetro de $2 \mathrm{~mm}$ no espaço imediatamente anterior ao distribuidor para garantir uma melhor distribuição do ar na coluna.

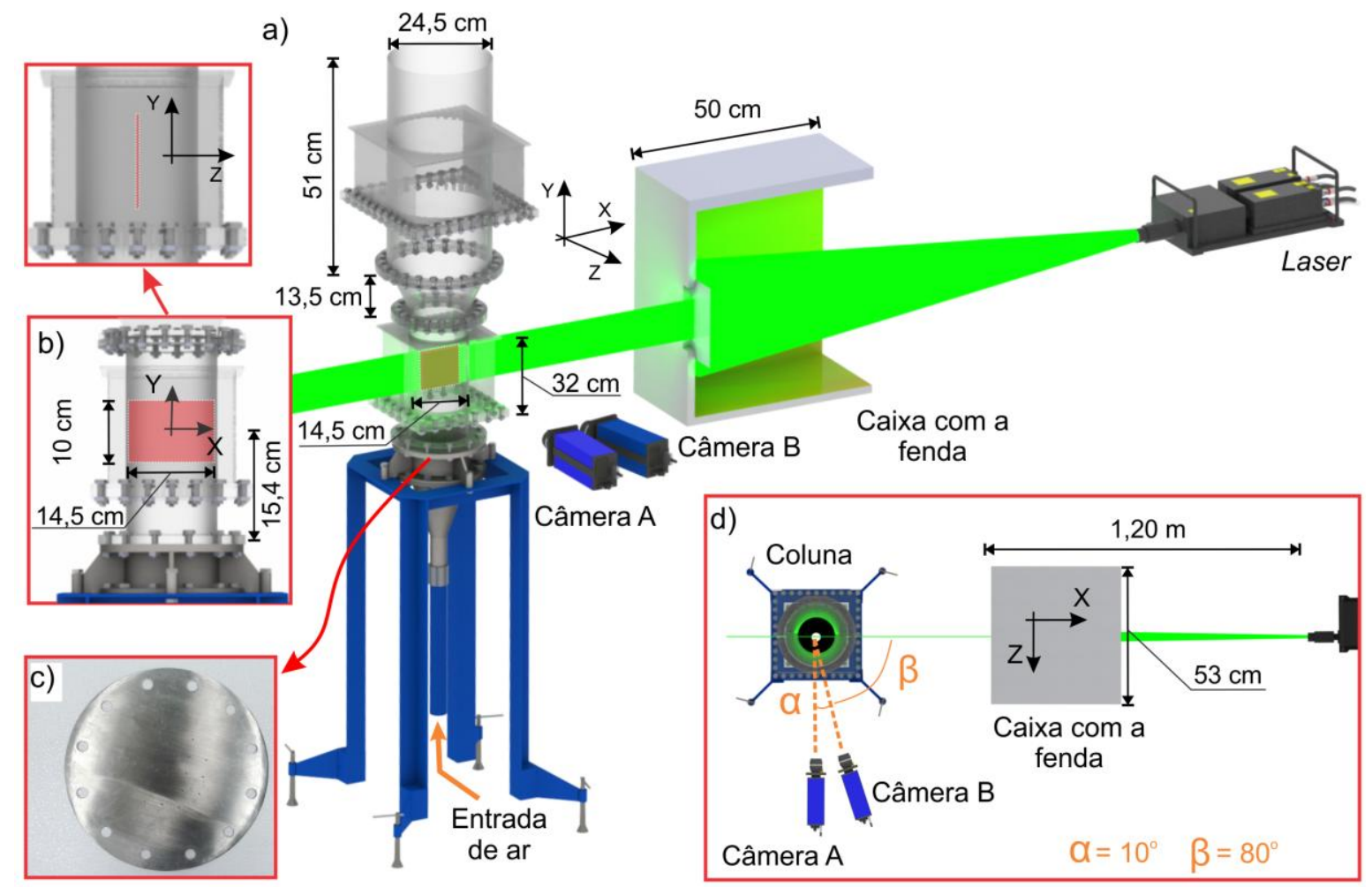

Figura 1 - (a) Coluna com expansão de diâmetro, sistema PIV e caixa com a fenda. (b) Área de investigação; (c) distribuidor da fase gasosa com 37 furos; (d) arranjo do sistema PIV na coluna.

Foi utilizado um sistema PIV com duas câmeras CCD (Charge Couple Device) e um sistema laser $\mathrm{Nd}$ :YAG $(200 \mathrm{~mJ} /$ pulso e $\lambda=532 \mathrm{~nm})$ desenvolvidos pela LaVision. A frequência de gravação foi de $4,92 \mathrm{~Hz}$ e foi utilizada Rodamina B (dp $=20-50 \mu \mathrm{m}$ e $\lambda=620$ $\mathrm{nm})$ como partícula traçadora. O sistema laser e as câmeras foram controlados por uma unidade sincronizadora interna (PTU-9) pelo software Davis 7.2. Para avaliar o melhor ângulo de gravação das partículas traçadoras, foram utilizadas as câmeras com um deslocamento angular de 90 (câmera A) e 80 (câmera B) graus em relação à folha de luz laser (Figura 1d). Cada câmera possui uma lente objetiva com distância focal de $60 \mathrm{~mm}$ modelo Nikon MicroNikorr $(f / 2.8 \mathrm{D})$ e um filtro passa-alta que permite apenas a passagem da luz com comprimento de onda próximo ao da Rodamina. Para obter uma espessura de luz definida $(1 \mathrm{~mm})$ e um perfil de intensidade do tipo Top-Hat, foi utilizada uma caixa com fenda localizada a 1,20 m 
do sistema laser. Além de utilizar uma caixa de acrílico com água na região investigada, foi feito um procedimento de calibração para diminuir os erros causados pela distorção da imagem na curvatura da coluna. A resolução do sistema de imagem foi de 24 pixels $/ \mathrm{mm}$. Depois de gravadas (do tipo double-frame), as imagens foram pré-processadas e o procedimento de correlação cruzada foi feito para determinação da distribuição da velocidade do líquido. No pré-processamento, foi aplicado um filtro RMS (roots mean square) 3 x 3 pixels. Na correlação cruzada, foi utilizada uma estratégia de interrogação com dois passos, sendo o primeiro com um tamanho de 128 pixels (sobreposição de $25 \%$ das janelas adjacentes) e o segundo com 64 pixels (sobreposição de $50 \%$ das janelas adjacentes). No pósprocessamento, foi usado o teste da mediana de Westerweel e Scarano (2005) com um limite de remoção igual a 2 e uma vizinhança de $3 \times 3$ pixels. A Tabela 1 apresenta os principais parâmetros utilizados do sistema PIV.

Tabela 1 - Principais parâmetros utilizados do sistema PIV

\begin{tabular}{|l|l|}
\hline Tempo entre o frame 0 e 1(dt) & $1500 \mu \mathrm{s}$ \\
\hline Deslocamento máximo da imagem de partícula & $15-20$ pixels \\
\hline Frequência de gravação & $4,92 \mathrm{~Hz}$ \\
\hline Tamanho do pixel & $6,45 \mu \mathrm{m}$ \\
\hline Diâmetro da partícula traçadora (Rodamina B) & $20-50 \mu \mathrm{m}$ \\
\hline Potência do laser & $35 \%$ \\
\hline Espessura da folha de luz & $1 \mathrm{~mm}$ \\
\hline Abertura da lente (f\#) & 2.8 \\
\hline Diâmetro da imagem de partícula & $2-3$ pixels \\
\hline Resolução do sistema de imagem (Câmera A e B) & $\sim 24$ pixels $/ \mathrm{mm}$ \\
\hline Tamanho da área investigada & $14,5 \times 10 \mathrm{~cm}$ \\
\hline Erro da calibração (Câmera A e B) & 0,580 e 0,573 pixel \\
\hline
\end{tabular}

Foram avaliadas a qualidade das medidas PIV pra uma vazão de ar de igual a $2 \mathrm{~L} / \mathrm{min}, 5$ $\mathrm{L} / \mathrm{min}, 10 \mathrm{~L} / \mathrm{min}, 15 \mathrm{~L} / \mathrm{min}$ e $20 \mathrm{~L} / \mathrm{min}$ analisando o espalhamento da luz na coluna e valor de correlação dos campos de velocidade obtidos.

\section{RESULTADOS E DISCUSSÕES}

A Figura 2 apresenta o frame 0 e 1, sem tratamento, gravadas pelas câmeras A e B para uma vazão de gás de $2 \mathrm{~L} / \mathrm{min}$. Percebe-se que a intensidade de luz no frame 0 é menor que a do frame 1 para as duas câmeras. O frame 0 e 1 da câmera A (Figura $2 \mathrm{a}$ e $2 \mathrm{~b}$ ) apresenta uma intensidade média de 67,98 e 89,12 counts, respectivamente. Essa diferença pode ser explicada pela interferência do primeiro pulso de luz laser que se espalha no ambiente e aumenta a quantidade de ruído no frame 1. A intensidade dos frames da câmera B é menor do que o da câmera A. O frame 0 e 1 da câmera B (Figura $2 \mathrm{c}$ e $2 \mathrm{~d}$ ) apresenta uma intensidade média de 46,45 e 69,64 counts, respectivamente. Mesmo com o uso do filtro passa-alta nas câmeras, a luz espalhada pelas bolhas interfere na intensidade de fundo dificultando a identificação da imagem de partícula. A Figura 3 apresenta o perfil de intensidade média de 2500 imagens em $\mathrm{Y}=15,4 \mathrm{~cm}$ para uma vazão de gás igual a $5 \mathrm{~L} / \mathrm{min}, 10 \mathrm{~L} / \mathrm{min}, 15 \mathrm{~L} / \mathrm{min}$ e $20 \mathrm{~L} / \mathrm{min}$. 


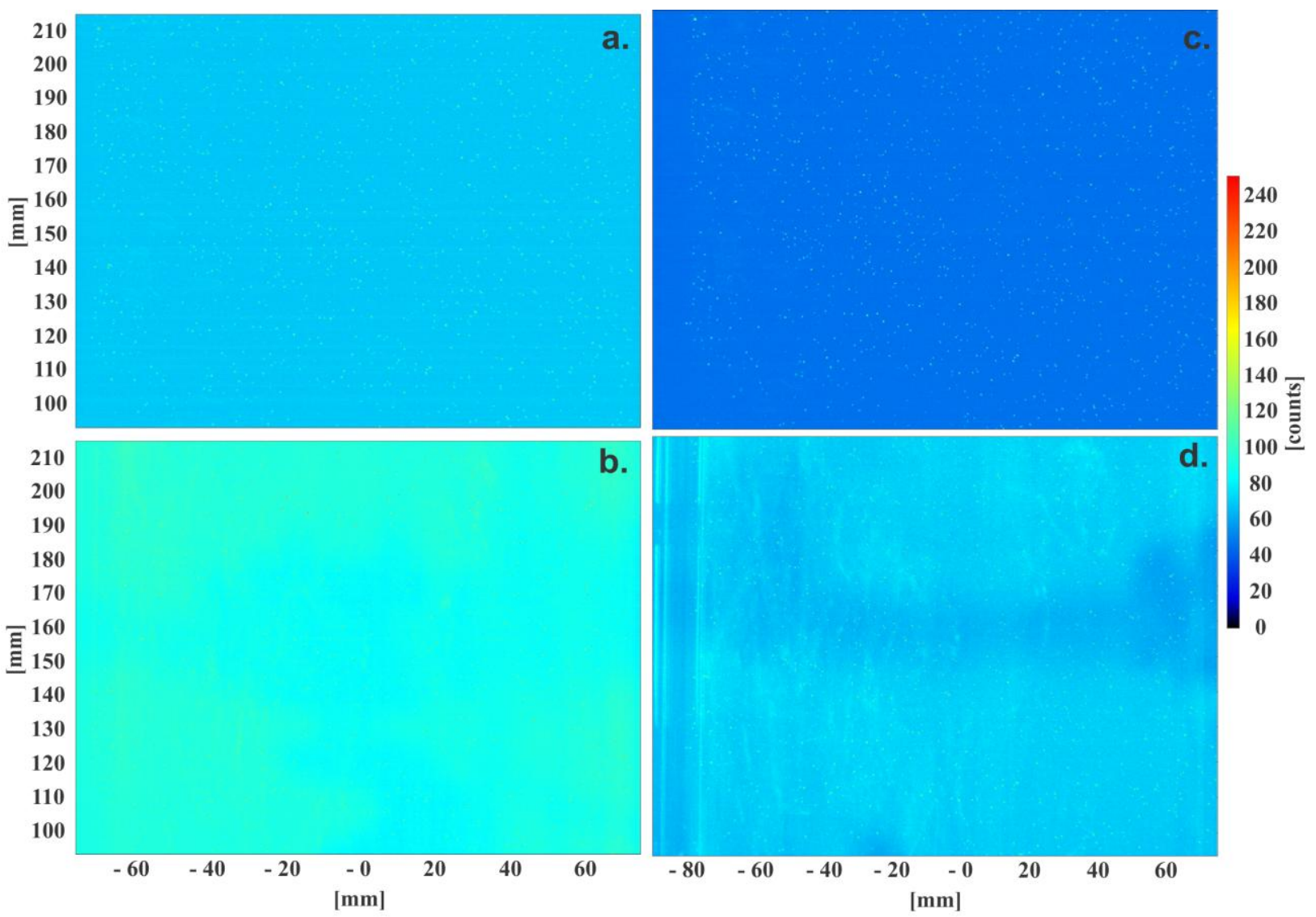

Figura 2 - Frame 0 e 1 sem tratamento gravados pelas câmeras A (a-b) e B (c-d) para uma vazão de gás de $2 \mathrm{~L} / \mathrm{min}$.

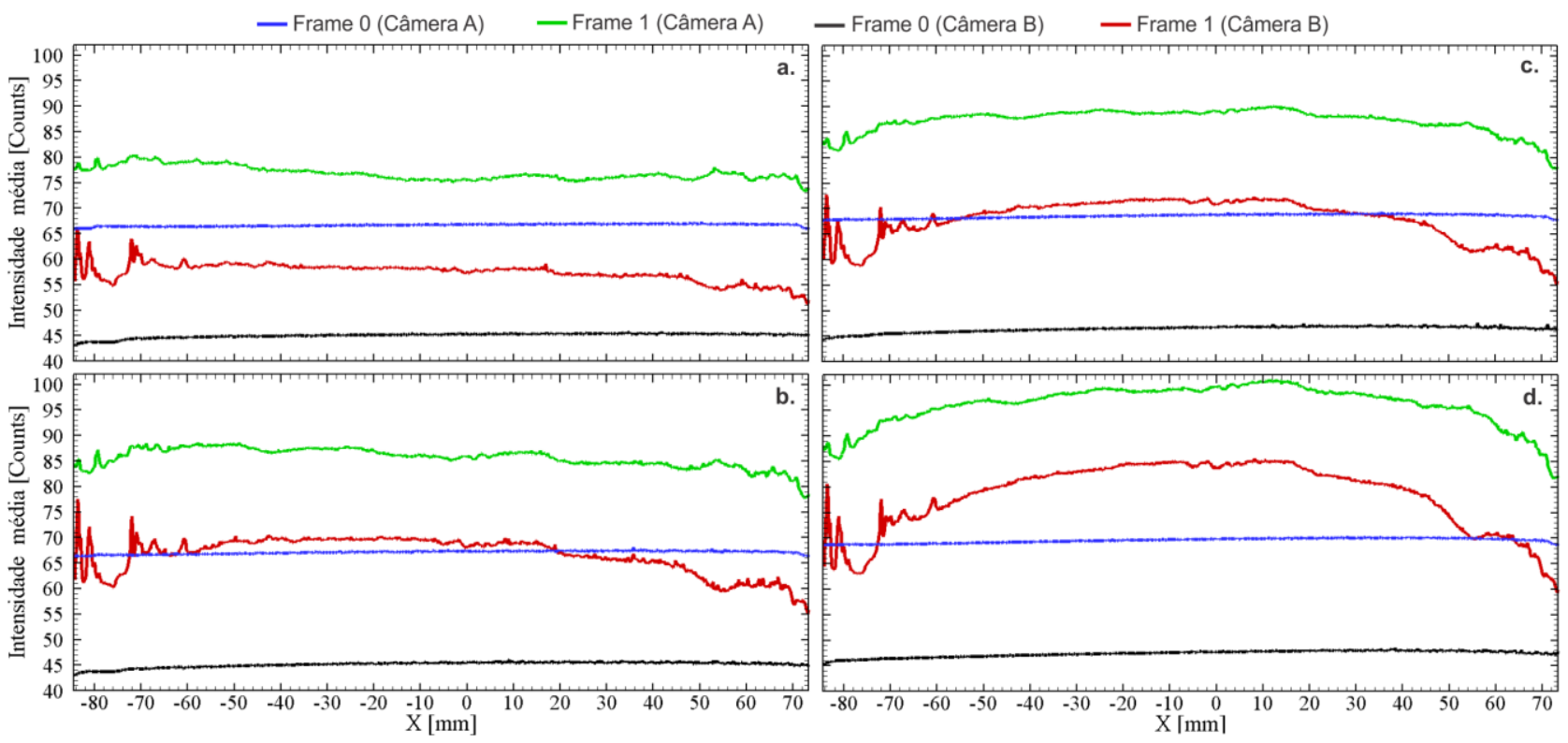

Figura 3 - Perfil de intensidade média de 2500 imagens em $\mathrm{Y}=15,4 \mathrm{~cm}$ para uma vazão de gás igual a (a) $5 \mathrm{~L} / \mathrm{min}$, (b) $10 \mathrm{~L} / \mathrm{min}$, (c) $15 \mathrm{~L} / \mathrm{min}$ e (d) $20 \mathrm{~L} / \mathrm{min}$. 
Percebe-se na Figura 3 que a intensidade do frame 0 das câmeras A e B se manteve constante com o aumento da vazão de gás, enquanto que a intensidade do frame 1 aumentou no centro da coluna. Esse aumento de intensidade no centro da coluna pode ser explicado pela maior presença de bolhas nessa região. A intensidade do frame 1 da câmera A foi maior que a câmera B para todas as vazões. Pela análise da Figura 2 e Figura 3, a câmera B é a melhor opção para determinação do campo de velocidade por PIV na coluna de bolhas em relação à câmera A. Foi utilizado o estudo feito por Broder e Sommerfeld (2002), que analisou o melhor ângulo de gravação das imagens de partículas de Rodamina B em uma coluna de bolhas, para a escolha do ângulo de 80 graus da câmera B.

A Figura 4 apresenta o perfil de intensidade média de 2500 imagens (equivalente a 8,4 min de experimento) após o pré-processamento do frame 0 e $1 \mathrm{em} \mathrm{Y}=15,4 \mathrm{~cm}$ em função da vazão de gás. No frame 0 e 1 (Figura 4a) em Y $=15,4 \mathrm{~cm}$, o perfil da intensidade média é aproximandamente o mesmo para as vazões de $2,5,10$ e $15 \mathrm{~L} / \mathrm{min}$. A intensidade média para o frame 0 foi de 1,$45 ; 1,47 ; 1,36 ; 1,25$ e 1,92 count para as vazões de $2,5,10,15$ e $20 \mathrm{~L} / \mathrm{min}$, respectivamente. A intensidade média para oframe 1 foi um pouco maior que oframe 0: 2,63; 2,$06 ; 2,44 ; 2,33$ e 2,76 counts para as vazões de $2,5,10,15$ e $20 \mathrm{~L} / \mathrm{min}$, respectivamente.

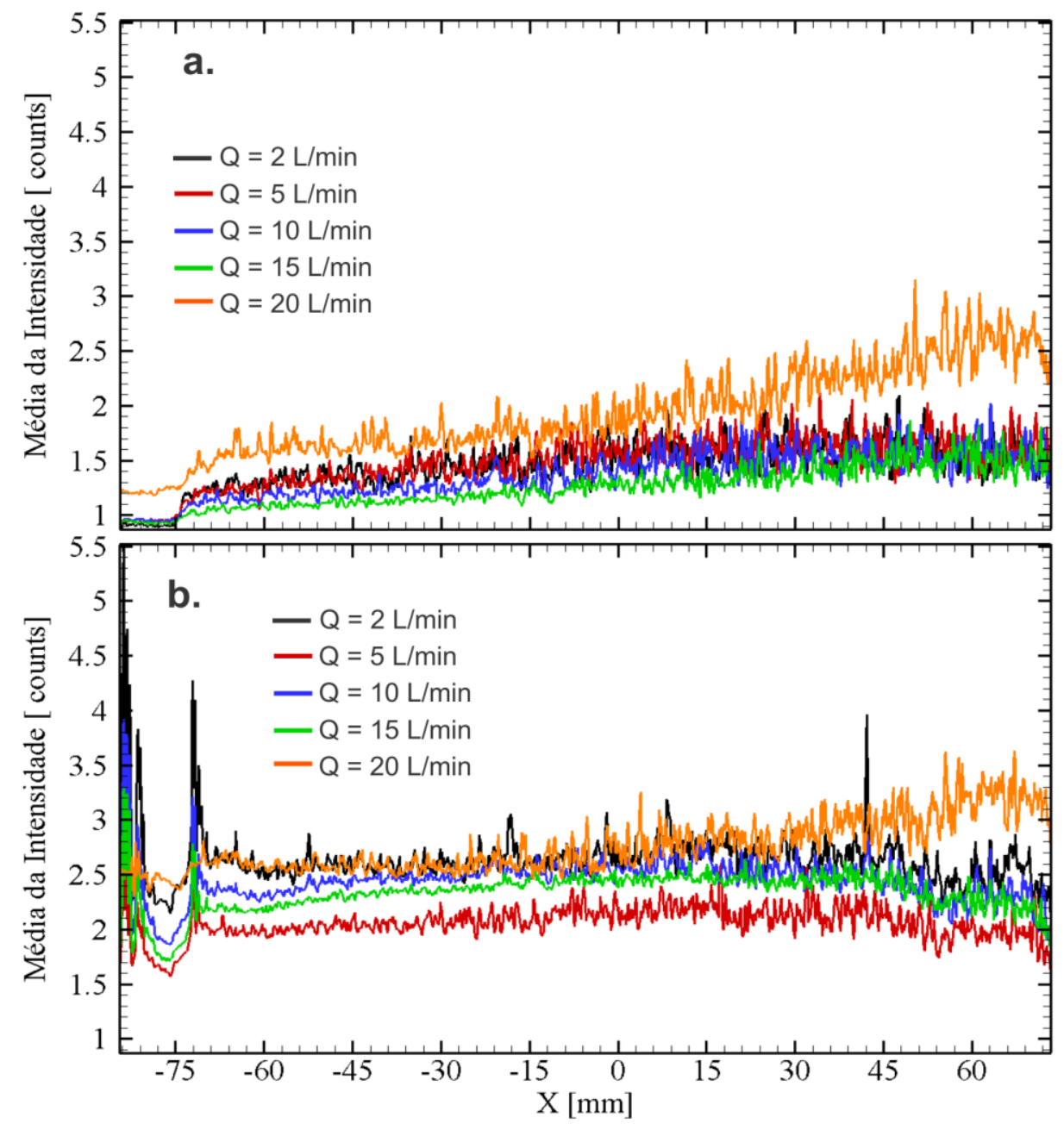

Figura 4 - Perfil de intensidade média de 2500 imagens após o pré-processamento do frame (a) 0 e (b) 1 em Y = 15,4 cm em função da vazão de gás. 
A Figura 5 corresponde aos perfis da velocidade média do líquido obtidos para diferentes vazões de entrada de gás. Observa-se que a velocidade axial é maior que a velocidade radial para todas as vazões. Para vazões mais baixas, percebe-se uma assimetria no perfil de Vy (Figura 5a). Para vazões mais altas, o perfil de Vy se aproxima de uma parábola (concavidade para baixo) com valores negativos próximos as paredes, produto da influência das bolhas em ascensão. Segundo Bröder e Sommerfeld, (2009) esta assimetria pode ser causada por perturbações na areação e pela natureza do escoamento dentro da coluna. Esse comportamento não ideal também pode ser relacionado ao projeto do distribuidor. O perfil $\mathrm{Vx}$ também apresenta uma simetria com o aumento da vazão de ar.
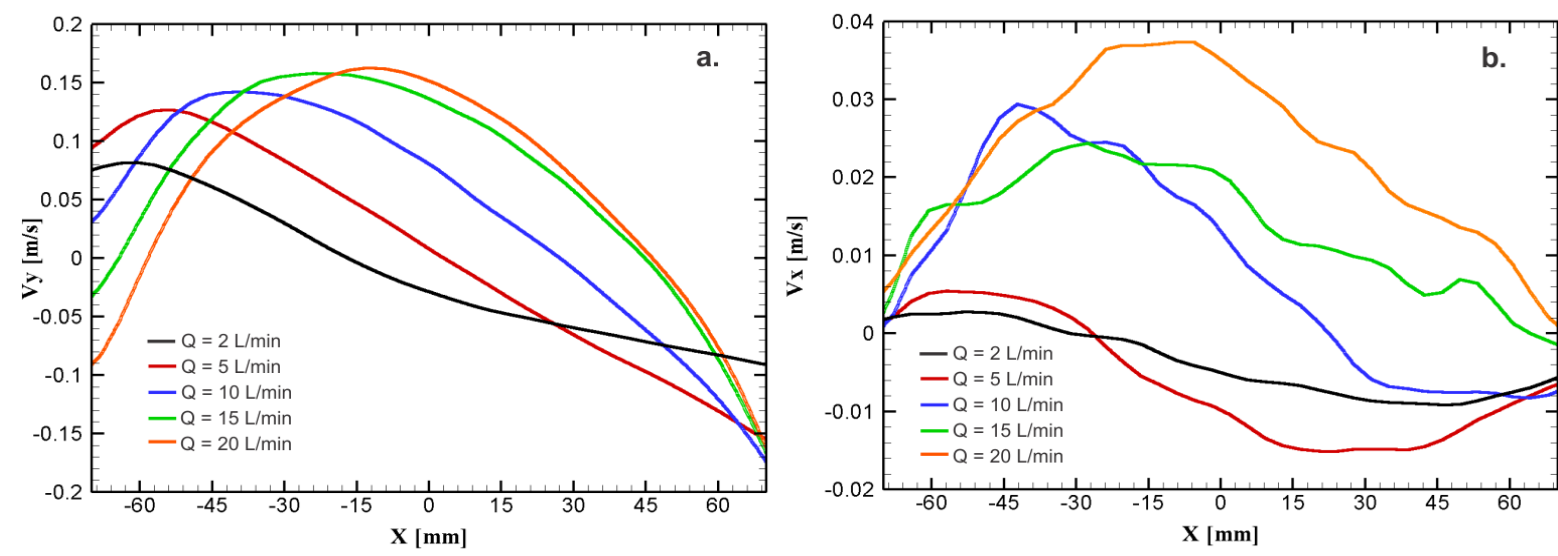

Figura 5 - Perfil da componente (a) Y e (b) X da velocidade do líquido para diferentes vazões de gás em $Y=15,4 \mathrm{~cm}$.

A Figura 6 apresenta o perfil da média de correlação de 2500 imagens em Y =15,4 cm e o histograma da média de correlação pra toda a área investigada em função da vazão de gás. Com o aumento da vazão, o valor de correlação diminui. Isso pode ser explicado pela perda de pares de imagens de partículas com o movimento para fora do plano de luz (direção Z), pela falta de iluminação na região contrária a posição do sistema laser $(-70 \mathrm{~mm}<\mathrm{X}<0 \mathrm{~mm})$ ou porque a luz espalhada pela Rodamina não consegue "sair" da coluna por conta das bolhas. $\mathrm{O}$ valor de correlação depende principalmente da quantidade de pares de imagem de partícula e dos seus movimentos coerentes (para a mesma direção) na área de interrogação. A média do valor de correlação para as 2500 imagens foi de 0,$68 ; 0,59 ; 0,57 ; 0,43$ e 0,40 para as vazões de 2, 5, 10, 15 e $20 \mathrm{~L} / \mathrm{min}$, respectivamente. Quanto menor o valor de correlação, maior a probabilidade de erro na detectibilidade de pico (escolha do deslocamento do pico mais alto) causado por ruído (picos secundários).

A Figura 7 apresenta a distribuição do valor de correlação para um instantâneo de velocidade para uma vazão de $20 \mathrm{~L} / \mathrm{min}$ e uma área de interrogação destacando os picos com valor de correlação de 0,2; 0,4 e 0,7. A linha branca tracejada na Figura 7a apresenta o erro no cálculo do campo de velocidade causado principalmente pela presença de bolhas. Além desse erro, percebe-se um valor de correlação de 0,2 e 0,4. A velocidade de ascensão do líquido causada pela presença de bolhas pode ser encontrada nessa região e consequentemente o baixo valor de correlação. 

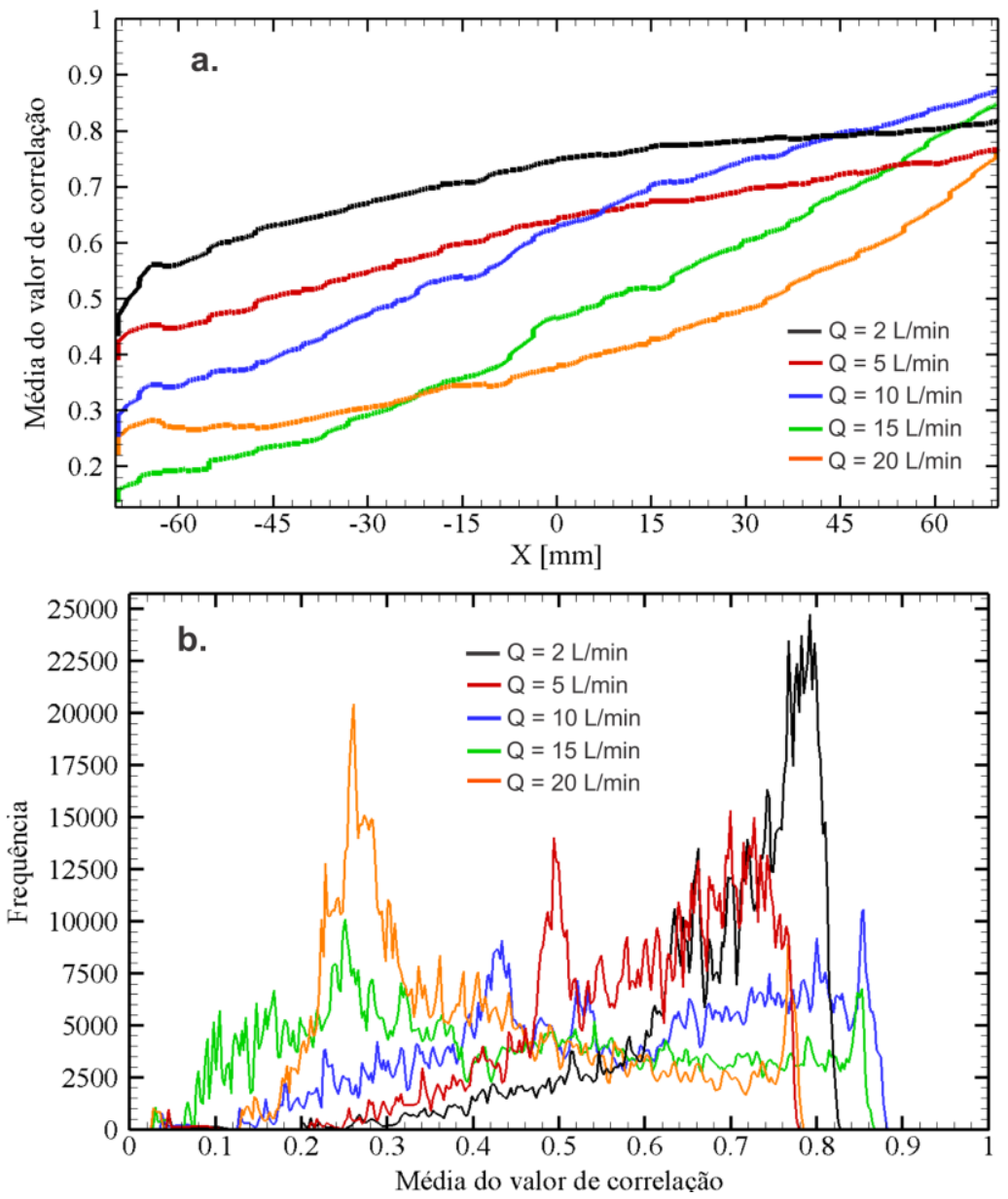

Figura 6 - (a) Perfil da média de correlação de 2500 imagens em Y = 15,4 cm e (b) histograma da média de correlação pra toda a área investigada em função da vazão de gás.

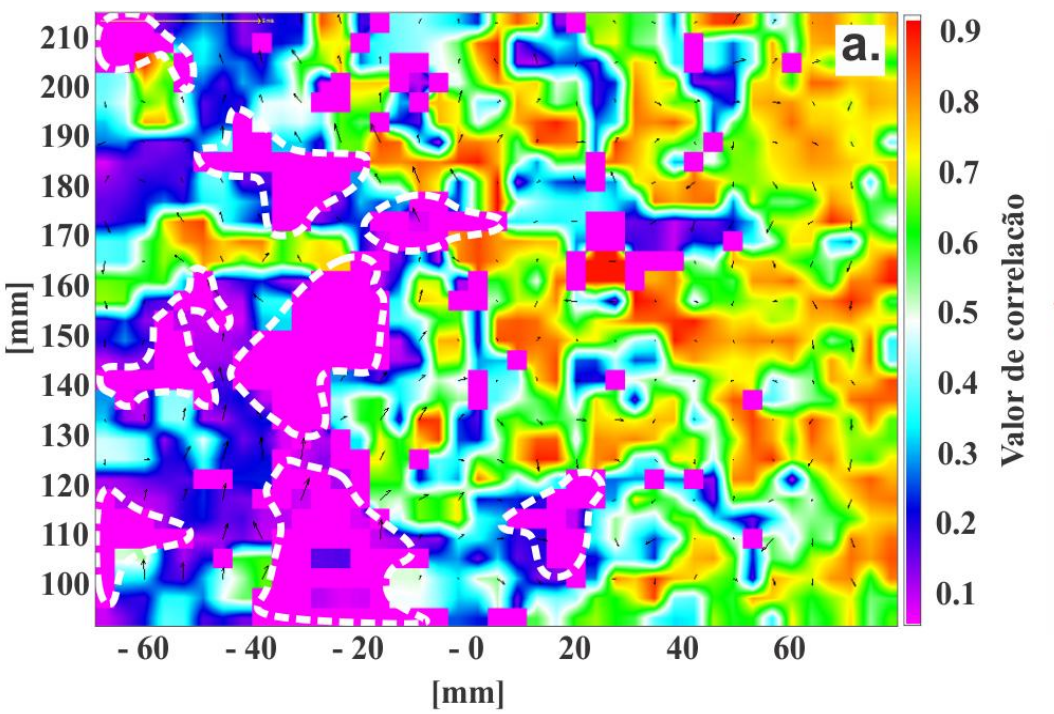

b.

Figura 7 - (a) Distribuição do valor de correlação para um instantâneo de velocidade para uma vazão de 20L/min e uma (b) área de interrogação destacando os picos com valor de correlação de 0,$2 ; 0,4$ e 0,7 . 


\section{CONCLUSÃO}

Esse trabalho avaliou a qualidade a qualidade das medidas PIV para uma vazão de ar de igual a $2 \mathrm{~L} / \mathrm{min}, 5 \mathrm{~L} / \mathrm{min}, 10 \mathrm{~L} / \mathrm{min}, 15 \mathrm{~L} / \mathrm{min}$ e $20 \mathrm{~L} / \mathrm{min}$ analisando o espalhamento da luz na coluna e valor de correlação dos campos de velocidade obtidos. Foram analisados dois arranjos do sistema de gravação e a câmera com um deslocamento angular de 80 graus, em relação ao plano de luz laser, apresentou a menor quantidade de iluminação de fundo (ruído). Com o aumento da vazão, o valor médio de correlação para 2500 imagens diminui principalmente em regiões de alta concentração de bolhas. Além disso, os resultados mostram um perfil de Vy assimétrico à medida que a vazões de gás diminui.

\section{AGRADECIMENTOS}

Os autores agradecem ao apoio financeiro da Petróleo Brasileiro S. A. - PETROBRÁS, termo de compromisso 0050.0070828.11.9, que garantiu o desenvolvimento deste trabalho e à CAPES.

\section{REFERÊNCIAS}

BRÖDER, D.; SOMMERFELD, M. An advanced LIF-PLV system for analysing the hydrodynamics in a laboratory bubble column at higher void fraction. Experiments in Fluids. v. 33, p 826. 2002.

BRÖDER, D.; SOMMERFELD, M. Planar Shadow image velocimetry for the analysis of the hydrodynamics in bubble flows. Meas. Sci. Technol. v. 18, p. 2513-2528, 2007.

PRASAD, A. Particle image velocimetry-Review article. Current Science, v. 79, p. 51-60, 2000.

WESTERWEEL, J.; SCARANO, F. Universal outlier detection for PIV data. Experiments in Fluids, v. 39, n. 6, p. 1096-1100, 2005.

SOMMERFELD, M.; BRÖDER, D. Analysis of Hydrodynamics and Microstructure in a Bubble Column by Planar Shadow Image Velocimetry. Experiments in Fluids. v. 48, pp 330-340. 2009.

WESTERWEEL, J.; SCARANO, F. Universal outlier detection for PIV data. Experiments in Fluids, v. 39, n. 6, p. 1096-1100, 2005. 